\title{
O consumo de álcool em serviços de saúde mental no Rio Grande do Sul ${ }^{1}$
}

\author{
Luciane Prado Kantorski ${ }^{a}$, Vanda Maria da Rosa Jardim ${ }^{a}$, Michele Mandagará de Oliveira ${ }^{a}$, \\ Poliana Farias Alves ${ }^{\mathrm{b}}$, Karine Langmantel da Silveira ${ }^{\mathrm{a}}$, \\ Carin Vieira Weiss ${ }^{\mathrm{c}}$, Cássia Vieira Weiss ${ }^{\mathrm{c}}$
}

\author{
${ }^{\text {a}}$ Faculdade de Enfermagem, Universidade Federal de Pelotas - UFPEL, Pelotas, RS, Brasil \\ bPrograma de Pós Graduação, Faculdade de Enfermagem, Universidade Federal de Pelotas - UFPEL, \\ Pelotas, RS, Brasil \\ ${ }^{c}$ Curso de Educação Física, Universidade Federal de Pelotas - UFPEL, Pelotas, RS, Brasil
}

\begin{abstract}
Resumo: Introdução: Segundo a Organização Mundial de Saúde, em 2004, aproximadamente 76,3 milhões de pessoas apresentaram problemas com o uso do álcool e o alcoolismo era uma das maiores preocupações de saúde pública. Portanto, os riscos decorrentes da associação de transtornos psiquiátricos com o uso de álcool devem ser considerados no contexto dos serviços de saúde mental. Objetivo: Analisar o consumo de álcool dos usuários de Serviço Residencial Terapêutico (SRT) e do Centro de Atenção Psicossocial (CAPS) no Rio Grande do Sul. Metodologia: O estudo é um recorte da pesquisa REDESUL - Redes que Reabilitam, de setembro a dezembro de 2009, em cinco municípios do Rio Grande do Sul. A amostra total foi de 392 usuários, desses, 143 eram do SRT e 270 eram dos CAPS, com uma intersecção de 21 usuários. Resultados: Os resultados mostraram que dos 392 usuários dos SRT e CAPS, apenas 29 tinham consumido bebida alcoólica nas últimas quatro semanas. Desses 29, a maioria apresentou idade entre 31 e 59 anos, era do sexo masculino, solteiro e apenas $n=13(48,28 \%)$ relataram conhecer seu transtorno psiquiátrico, havendo prevalência da esquizofrenia $n=7(24,13 \%)$, seguida dos transtornos bipolares $n=3(10,34 \%)$. Conclusão: É necessário que as equipes de saúde mental estejam capacitadas para atuar também com os usuários de álcool, independente do tipo de serviço de saúde mental em que atuem, e que elas desenvolvam ações em relação à orientação sobre o consumo do álcool, a adesão ao tratamento, a reabilitação e a inserção dos usuários na comunidade.
\end{abstract}

Palavras-chave: Saúde Mental. Alcoolismo. Serviços de Saúde Mental.

\section{Consumption of alcohol in mental health services in Rio Grande do Sul state, Brazil}

\begin{abstract}
Introduction: Alcoholism has been a major concern of public health worldwide. According to the World Health Organization (WHO), approximately 76.3 million people presented problems of alcohol abuse in 2004. Therefore, the risks arising from the association of psychiatric disorders with alcohol consumption should also be considered in the context of mental health services. Objective: This study aimed to analyze alcohol consumption by the users of Therapeutic Residential Services - SRT and Psychosocial Care Centers - CAPS in five municipalities in the state of Rio Grande do Sul, Brazil. Methodology: The present study is part of a research entitled Rehabilitation Networks - REDESUL, carried out from September to December 2009 in five municipalities of the aforementioned Brazilian state. The total sample comprised 392 users: 143 from the SRT and 270 from the CAPS services, with intersection of 21 members. Results: The results showed that of the 392 care service users, only 29 had consumed alcohol during the four weeks prior to the survey. The majority of these 29 users were between 31 and 59 years
\end{abstract}

Autor para correspondência: Poliana Farias Alves, Faculdade de Enfermagem, Universidade Federal de Pelotas, Rua Gomes Carneiro, 1, Centro, CEP 96010-610, Pelotas, RS, Brasil, e-mail: polibrina@hotmail.com

Recebido em 31/8/2013; Revisão em 20/2/2014; Aceito em 4/5/2014. 
old, male, single, and only $\mathrm{n}=13(48.28 \%)$ reported being aware of their psychiatric disorders, with prevalence of schizophrenia $n=7(24.13 \%)$ followed by bipolar disorders $n=3(10.34 \%)$. Conclusion: It is necessary that the mental health teams are also trained to work with alcohol users, regardless of the type of mental health service they work for, and that they develop actions in relation to guidance on alcohol consumption, treatment adherence, rehabilitation, and integration of users to the community.

Keywords: Mental Health. Alcoholism. Mental Health Services.

\section{Introdução}

A Política Nacional de Saúde Mental, apoiada na lei 10.216/01, busca consolidar um modelo de atenção à saúde mental aberto e de base comunitária que garanta a livre circulação de pessoas com transtornos mentais pelos serviços, comunidades e cidades. Esse modelo conta com uma rede de serviços, incluindo os Centros de Atenção Psicossocial (CAPS) e os Serviços Residenciais Terapêuticos (SRT) (BRASIL, 2004).

No Brasil, os CAPS e os SRT são serviços de Saúde Mental que têm como proposta a materialização dos ideais sanitários e da Reforma Psiquiátrica como garantia de acesso, acolhimento e tratamento dignos ao indivíduo com sofrimento mental, na comunidade onde está inserido. Os CAPS, nesse sentido, aparecem como instrumentos que possibilitam reorganizar a rede de saúde mental brasileira, pois se acredita que náo basta tratar as pessoas como indivíduos em sofrimento, elas precisam ser tratadas como cidadãs, que têm o mesmo direito ao acesso e aos serviços, assim como de atendimento nos diferentes níveis de atenção/complexidade (PINHO; HERNANDÉZ; KANTORSKI, 2010).

Os CAPS são serviços substitutivos estratégicos que organizam a rede de atenção e cuidado em saúde mental. Eles devem ser territorializados, situados no espaço de convívio social, perto do círculo familiar, devendo esses serviços ter a capacidade de resgatar as potencialidades existentes na comunidade e ser agentes de inclusão social e de reabilitação psicossocial (PUEL; HECKRATH; RIESINGER, 2006).

Considerando a efetividade dessa rede de serviços substitutivos, o Serviço Residencial Terapêutico (SRT) foi um dispositivo que surgiu para acolher os egressos de hospitais psiquiátricos, que muitas vezes não possuíam um suporte social e/ou familiar, ou que não tinham condiçóes de moradia (SILVA; AZEVEDO, 2011).

Segundo Pinho et al. (2009), para que a função da Reabilitação Psicossocial seja possível e de qualidade na atenção aos usuários de álcool e outras drogas são necessárias discussōes sobre as questôes associadas às variáveis reais, ou seja, o serviço de Reabilitação Psicossocial, o significado do tratamento em si; os recursos disponíveis (humanos comunitários e materiais) e o contexto de vida do indivíduo.

Porém, enquanto o modelo de atençáo psicossocial, oriundo da Reforma Psiquiátrica, não é plenamente assumido pelo sistema de saúde mental no país, as consequências negativas recaem principalmente sobre os usuários e seus familiares. Há de se assumir também que existe entendimento insuficiente desse modelo de atenção entre os trabalhadores dos serviços de saúde mental (GURGEL; MOCHEL; CARVALHO FILHA, 2010).

É importante considerar no contexto do atendimento ao usuário portador de transtorno psiquiátrico os riscos oriundos da associação com o uso de álcool. O alcoolismo atinge todas as classes sociais, em diferentes idades, sem escolha de cor ou sexo. As bebidas alcoólicas existem desde a antiguidade, como a cerveja no Egito e na Mesopotâmia e como o vinho na Grécia. A palavra alcoolismo foi usada pela primeira vez no século XIX; desde então popularizou-se de tal forma que, atualmente, além de significar um fenômeno médico-social, tem a conotação de rótulo estigmatizante (JORGE et al., 2007).

Segundo as informações da Organização Mundial da Saúde (2004), em 2004 existiam aproximadamente 2 bilhóes de pessoas no mundo consumidoras de bebidas alcoólicas e 76,3 milhóes apresentam problemas com o uso do álcool.

O alcoolismo tem sido uma das maiores preocupaçóes da saúde pública no mundo, estando associado a diversos outros problemas como: mortes no trânsito, desentendimentos familiares e afetivos e separação de casais, sendo, também, relatado em casos de homicídio, espancamento de crianças e de mulheres, deserção do trabalho, da escola, entre outros (NASCIMENTO; JUSTO, 2000).

Diversos estudos, principalmente na Europa e nos Estados Unidos da América (EUA), relatam os efeitos negativos do uso/dependência de substâncias psicoativas entre pacientes com transtornos mentais, tentando estabelecer as potenciais diferenças entre pacientes que abusam de álcool ou substâncias psicoativas, principalmente nas implicaçóes quanto a diagnóstico, tratamento e prognóstico. Há evidências 
de que mesmo o uso infrequente e de pequenas doses de drogas, legais ou ilegais, pode levar o indivíduo com transtornos mentais graves a consequências mais sérias do que as vistas na população em geral e está associado a mais efeitos negativos ligados aos transtornos mentais (ZALESKI et al., 2006).

Por isso, o presente estudo tem por objetivo analisar o consumo de álcool dos usuários de SRT e CAPS em cinco municípios do estado do Rio Grande do Sul.

\section{Metodologia}

O presente estudo é um recorte da pesquisa intitulada REDESUL - Redes que Reabilitam (KANTORSKI, 2011), realizado no período de setembro a dezembro de 2009. Para a definição da amostra assumiu-se como marcador de rede de serviços em saúde mental a existência dos serviços tipo SRT e CAPS II. Assim foram identificados cinco municípios do Rio Grande do Sul, sendo eles: Alegrete, Bagé, Caxias do Sul, Porto Alegre e Viamão. A amostra total de entrevistados foi de 392 usuários, desses, 143 eram usuários do SRT e 270, dos CAPS, com uma intersecção de 21 usuários que utilizavam concomitantemente os dois serviços. O projeto foi encaminhado ao Comitê de Ética da Faculdade de Odontologia da Universidade Federal de Pelotas, tendo sido aprovado sob o parecer de n. 073/2009.

Foi realizado um estudo transversal de experiências inovadoras de composiçáo de redes através de uma abordagem epidemiológica. Para tal, foi utilizado um questionário estruturado aplicado aos usuários e, em situações em que o entrevistado não apresentava condiçôes de respondê-lo, direcionado ao cuidador. Utilizaram-se dados do perfil e um recorte do Alcohol Use Disorders Identification Test (AUDIT): esse questionário foi elaborado pela Organização Mundial da Saúde (BABOR et al., 2001) e validado no Brasil por Méndez em 1999. O instrumento é composto por 10 questóes sobre o uso do álcool que se referem ao último ano. Utilizaram-se nesta pesquisa as quatro questóes finais do AUDIT, que se referem a problemas recentes na vida relacionados ao consumo (BERGMAN; KÄLLMÉN, 2002).

Para a análise dos dados utilizou-se o software EPI-INFO e eles passaram por dupla digitação por digitadores independentes no software EPI - INFO e STATA. A limpeza dos dados ocorreu por comparaçáo dos dois arquivos e avaliaçáo dos erros de amplitude e consistência. A base de dados foi utilizada para as correções necessárias. O controle de qualidade foi realizado através da checagem de cada entrevistador, ao final da entrevista, e também na replicação de $10 \%$ das entrevistas realizadas.

\section{Resultados}

Foram realizadas entrevistas com 392 usuários, sendo 205 usuários do sexo masculino e 187 usuárias do sexo feminino. Considerando-se apenas o perfil dos 29 usuários de SRT e CAPS que consomem bebida alcoólica podemos observar os dados que se apresentam na tabela a seguir:

Tabela 1. Perfil dos 29 usuários de CAPS e SRT que referiram ter feito uso de álcool nas últimas quatro semanas, RS, 2011.

\begin{tabular}{lcc}
\hline \multicolumn{1}{c}{ Idade } & N & $\%$ \\
\hline 19-30 anos & 6 & 20,69 \\
$31-59$ anos & 21 & 72,41 \\
$>$ que 60 anos & 1 & 3,45 \\
Não soube & 1 & 3,45 \\
Sexo & & \\
Feminino & 11 & 37,90 \\
Masculino & 18 & 62,10 \\
Estado Civil & & \\
Solteiro & 17 & 58,63 \\
Casado/Companheiro & 8 & 27,58 \\
Separado & 1 & 3,45 \\
Viúvo & 3 & 10,34 \\
Divorciado & 0 & 0 \\
\hline Fon: REDESUL (2011). & & \\
\hline
\end{tabular}

Fonte: REDESUL (2011).

Segundo a Tabela 1 ficou evidenciado que o maior índice de usuários de álcool concentra-se na faixa etária 31-59 anos, do sexo masculino e solteiro.

Quando especificado o uso de álcool nas últimas quatro semanas pelos usuários do Serviço Residencial Terapêutico, obteve-se que $\mathrm{n}=14(9,79 \%)$ usuários referiram ter consumido e $\mathrm{n}=129(90,1 \%)$ usuários referiram náo ter utilizado, mostrando que grande parte deles náo faz uso regular de bebida alcoólica.

Já os 270 usuários de CAPS foram classificados como semi-intensivos e intensivos. Referente aos semiintensivos, $\mathrm{n}=7(5,15 \%)$ usuários referiram ter feito uso de bebida alcoólica nas últimas quatro semanas e $\mathrm{n}=129(94,8 \%)$ referiram não ter consumido. Em relação aos intensivos, $\mathrm{n}=9(6,72 \%)$ usuários referiram ter consumido bebida alcoólica nas últimas quatro semanas, enquanto $\mathrm{n}=125(93,28 \%)$ usuários referiram não ter feito o consumo de bebida alcoólica.

Analisando-se a tabela acima fica evidenciado que poucos usuários dos CAPS e SRT das cidades 
entrevistadas utilizavam álcool (Tabela 2). Em Alegrete e Bagé são dois usuários em cada; em Caxias do Sul e Porto Alegre foram encontrados nove usuários em cada e em Viamão, sete dos entrevistados. Já em relação aos que consomem, a maioria náo sente culpa pelo seu consumo, nunca tiveram apagóes da noite anterior por causa da bebida e não se machucaram nem prejudicaram ninguém pelo seu consumo.

Analisando-se a Tabela 3 ficou evidenciado que dentre os usuários de álcool que relataram conhecer seu transtorno psiquiátrico, o que prevaleceu foi a esquizofrenia com $n=7(24,13 \%)$, seguida dos transtornos bipolares $\mathrm{n}=3(10,34 \%)$. Entretanto, há que se considerar que $\mathrm{n}=15(51,72 \%)$ dos 29 usuários que consumiam álcool não falaram, ou não conheciam seu diagnóstico.

\section{Discussão}

No estudo de Pillon e Luis (2004) evidencia-se a afirmação de que no contexto do modelo psicossocial de intervenção, fatores sociológicos tais como o desemprego, a privação social e outros podem ter efeitos importantes sobre o início e o uso continuado da substância pelo indivíduo. Também sexo, idade, religião, etnicidade, condiçôes socioeconômicas e ambiente familiar influenciam diretamente na opção individual de usar ou não a substância.

O alcoolismo é considerado uma doença de causas variadas, entretanto apresenta fatores primordiais como hereditariedade, os convívios sociais e familiares, independentemente de sexo e do nível social em que o indivíduo está inserido. É um hábito estimulado socialmente, tornando-se banal e característico de algumas sociedades (BRASIL, 2008).

Em uma pesquisa nacional realizada pelo CEBRID - Centro Brasileiro de Informaçôes Sobre Drogas, em 2006, estima-se que o uso de álcool no país fosse de $74,6 \%$ e a dependência estipulou-se em $12,3 \%$ da população brasileira. O menor uso de álcool ocorreu na regiāo Norte, $53,9 \%$, na região Sul, o índice de usuários de álcool foi de $73,9 \%$ e o maior índice apareceu no Sudeste, 80,4\%.

A pesquisa supracitada mostrou que em todas as regióes observaram-se mais dependentes de álcool para o sexo masculino, o que vai ao encontro dos dados apresentados neste estudo (CEBRID, 2006).

De acordo com os estudos de Alves, Kessler e Ratto (2004) sobre álcool e transtornos psiquiátricos, em algum momento de sua vida, em torno de 50\% dos usuários com transtornos mentais graves podem desenvolver problemas relativos ao consumo de álcool/drogas, e no que se refere aos transtornos mentais é comum que os relacionados ao consumo de álcool coexistam com outras doenças psiquiátricas. Esses autores também ressaltam a importância dos profissionais de saúde estarem a par de novas técnicas de avaliação, de intervenção e de novos tratamentos baseados no modo psicossocial desenvolvidos para esse tipo de usuário.

Em relação às questóes do AUDIT, os resultados encontrados referentes à frequência com que sentiu culpa ou remorso por beber, vão ao encontro dos estudos de Peuker, Fogaça e Bizarro (2006) e de Campos et al. (2011), que referiram que os

Tabela 2. Referente ao consumo de álcool e percepção sobre o seu consumo dos 29 usuários (terceiro bloco do AUDIT - Prejuízos pelo uso do álcool), RS, 2011.

\begin{tabular}{lcr}
\hline \multicolumn{1}{c}{ Realizou consumo de álcool nas últimas quatro semanas } & $\mathbf{N}$ & \% \\
\hline Não & 363 & 92,60 \\
Sim & 29 & 7,40 \\
Frequência no último ano que sentiu culpa ou remorsos por beber & & \\
Nunca & 18 & 62,06 \\
Menos de uma vez ao mês & 3 & 10,34 \\
Uma vez ao mês & 2 & 6,90 \\
Uma vez na semana & 1 & 3,45 \\
Todos os dias & 5 & 17,24 \\
Frequência no último ano que não conseguiu lembrar-se o que ocorreu na & & \\
noite anterior por causa da bebida & 15 & \\
Nunca & 6 & 20,63 \\
Menos de uma vez ao mês & 1 & 3,45 \\
Uma vez ao mês & 2 & 6,89 \\
Uma vez na semana & & \\
\hline
\end{tabular}

Fonte: REDESUL (2011). 
Tabela 3. Prevalência dos transtornos psiquiátricos nos 29 usuários de álcool, RS, 2011.

\begin{tabular}{lcc}
\hline Transtornos psiquiátricos & $\mathbf{N}$ & $\%$ \\
\hline Esquizofrênicos & 7 & 24,13 \\
Bipolaridade & 3 & 10,34 \\
Depressão maior & 2 & 6,90 \\
Outros & 2 & 6,90 \\
Não informaram & 15 & 51,72 \\
\hline
\end{tabular}

Fonte: REDESUL (2011).

entrevistados na sua maioria nunca tiveram esses problemas. Porém a questão que se refere a terceiros se importarem ou pedirem para que parassem de beber se distancia, visto que nos dados encontrados na pesquisa dos supracitados autores a prevalência apareceu naqueles que nunca receberam essas intervençôes $(86,7 \%$ e $83,69 \%)$, já nesse estudo a predominância da resposta dos usuários foi que tiveram a intervenção no último ano $(60,85 \%)$.

Segundo Silva et al. (2009), até o momento, no Brasil são poucos os estudos que investigaram a comorbidade, embora haja indícios de que a associaçáo entre transtornos mentais e abuso/ dependência de álcool e/ou drogas seja um problema relevante. $\mathrm{O}$ autor cita uma pesquisa realizada nos EUA em que 37\% dos usuários de álcool apresentam um diagnóstico psiquiátrico. Já nos dados encontrados na pesquisa do presente estudo esse valor chega a 48,27\% de usuários com comorbidade.

A esquizofrenia concomitante com o uso do álcool apareceu em 24,13\% dos entrevistados. Segundo Ramos et al. (1997), em relação às características sintomatológicas, pacientes esquizofrênicos com abuso de álcool apresentaram mais sintomas alucinatórios e delirantes, enquanto os pacientes com abuso em outras drogas apresentaram mais isolamento social, preocupações místicas e delírios de influência. E, segundo Zaleski et al. (2006), os indivíduos com esquizofrenia e com abuso de substâncias têm um prognóstico pior do que os pacientes com apenas um desses transtornos e são de difícil tratamento.

O álcool também pode produzir sintomas de depressão, ansiedade, agitação e hipomania/mania durante a intoxicação pelo uso (ALVES; KESSLER; RATTO, 2004).

Os dados desta pesquisa mostram que $6,90 \%$ dos usuários de álcool apresentam concomitantemente depressão. Já em relação à bipolaridade, 10,34\% dos usuários de bebidas alcoólicas apresentaram a comorbidade.

Segundo Ribeiro, Laranjeira e Cividanes (2005), entre os pacientes com problemas relacionados ao uso de álcool e drogas, um quarto apresenta algum transtorno do humor associado. Relatam também que sintomas depressivos podem predispor ao uso de substâncias químicas como o álcool.

A incidência de comorbidade com transtornos mentais graves parece estar aumentando. Uma das maiores dificuldades na abordagem do paciente com comorbidade está no diagnóstico diferencial, pois ocorre uma superposiçáo de sintomas. Um transtorno pode exacerbar ou mascarar o outro. Não é fácil, no início, estabelecer diferenças entre a presença de comorbidade psiquiátrica e abuso de substâncias psicoativas. Existe a hipótese de que os indivíduos com transtornos psiquiátricos acabam por usar o álcool como uma forma de automedicação, o que acaba por agravar o transtorno ansioso primário (ZALESKI et al., 2006).

O estudo também apresentou características importantes, pois vários usuários $\mathrm{n}=15(51,72 \%)$ consumidores de álcool não sabiam que tipo de transtorno os acometia. Pode-se sugerir que essa falta de informação talvez deva-se a falta de reconhecimento dos sinais e sintomas do transtorno pela equipe de saúde ou, ainda, ao não reconhecimento (aceitação) pelo próprio usuário da sua doença, seja por estigma ou por preconceito. Essas possibilidades, apesar de serem apenas hipóteses, são de alta relevância no que se refere à adesão ao tratamento, à reinserção do usuário e ao desenvolvimento de açóes que promovam uma mudança cultural na comunidade, pois se reconhece que esses são pontos constituintes de um novo modelo de atenção aos usuários dos serviços de saúde mental.

\section{Considerações}

Os resultados da pesquisa mostraram que num universo amostral de 392 usuários dos serviços SRT e CAPS apenas $\mathrm{n}=29(7,40 \%)$ usuários tinham consumido bebida alcoólica nas últimas quatro semanas e que dentre esses 29 a maioria apresentou-se em idade adulta e altamente produtiva. O consumo de bebida alcoólica no grupo estudado não apresentou significância estatística, ou seja, no grupo estudado existiam poucos consumidores de bebida alcoólica, entretanto há de se considerar que mesmo o álcool sendo uma substância licita, muitas pessoas podem ter vergonha de revelar o seu uso, independentemente da frequência do consumo. Além disso, há de se considerar também que muitos desses usuários possivelmente já teriam sofrido algum tipo de discriminação por serem portadores de transtornos psíquicos, assim assumir o uso de 
uma substância psicoativa poderia reforçar ainda mais o processo de exclusão social.

De acordo com os dados evidenciados, faz-se necessário que haja trabalhadores capacitados para atuar não somente com usuários acometidos por transtornos psiquiátricos mas também com aqueles que fazem consumo de bebidas alcoólicas. Que esses estejam engajados em produzir açôes de orientação e esclarecimento quanto ao uso prejudicial do álcool, quanto às interaçóes medicamentosas, informando sobre possíveis problemas decorrentes do uso concomitante do álcool com transtornos psiquiátricos, e em produzir açôes que promovam a superaçâo de estigmas e preconceitos, independentemente do serviço de saúde mental em que atuem.

Em uma esfera maior, também se percebe como necessário propor açôes referentes ao desenvolvimento de políticas públicas que reorientem a forma como o álcool é socialmente representado e que, apesar da sua licitude, as consequências negativas decorrentes do seu uso abusivo acarretam sérios prejuízos pessoais, econômicos e sociais.

\section{Referências}

ALVES, H.; KESSLER, F.; RATTO, L. R. C. Comorbidade: uso de álcool e outros transtornos psiquiátricos. Revista Brasileira de Psiquiatria, São Paulo, v. 26, p. 51-53, 2004. Suplemento 1.

BABOR, T. F. et al. AUDIT. The Alcohol Use Disorders Identification Test: guidelines for use in primary health care. Geneva: WHO, 2001.

BERGMAN, H.; KÄLLMÉN, H. Alcohol use among Swedes and psychometric evaluation of the alcohol use disorders identification test. Alcohol and Alcoholism, Oxford, v. 37, n. 3, p. 345-251, 2002.

BRASIL. Ministério da Saúde. Secretaria de Atenção à Saúde. Departamento de Ações Programáticas Estratégicas. Coordenação Geral de Saúde Mental. Saúde mental no SUS: os centros de atenção psicossocial. Brasília, 2004

BRASIL. Ministério da Saúde. Secretaria Nacional Anti Drogas. Prevenção ao uso indevido de drogas: curso de capacitação para conselheiros municipais. Brasília, 2008.

CAMPOS, J. A. D. B. et al. Consumo de álcool entre estudantes do ensino médio do município de Passos - MG. Ciência \& Saúde Coletiva, Rio de Janeiro, v. 16, n. 12, p. 4745-4754, 2011.

CENTRO BRASILEIRO DE INFORMAÇÓES SOBRE DROGAS PSICOTRÓPICAS - CEBRID. II Levantamento domiciliar sobre o uso de drogas psicotrópicas no Brasil. São Paulo: UNIFESP, 2006.

GURGEL, W. B.; MOCHEL, A. G.; CARVALHO FILHA, F. S. S. S. O abuso do álcool como problema político: análise das estratégias políticas de assistência ao consumidor abusivo de álcool no Brasil contemporâneo. Caderno de Pesquisa, São Luís, v. 17, n. 1, p. 61-68, 2010.
JORGE, M. S. B. et al. Alcoolismo nos contextos social e familiar: análise documental à luz de Pimentel. Rev RENE, Fortaleza, v. 8, n. 3, p. 34-43, 2007.

KANTORSKI, L. P. REDESUL - Redes que reabilitam: avaliando experiências inovadoras de composição de redes de atenção psicossocial. Pelotas, 2011.

MÉNDEZ, E. B. Uma versão brasileira do AUDIT (Alcohol Use Disorders Identificarion Test). 1999. $120 \mathrm{f}$. Dissertação (Mestrado)-Universidade Federal de Pelotas, Pelotas, 1999.

NASCIMENTO, E. C.; JUSTO, J. S. Vidas errantes e alcoolismo: uma questão social. Psicologia: Reflexão e Crítica, Porto Alegre, v. 13, n. 3, p. 529-538, 2000. http://dx.doi.org/10.1590/S0102-79722000000300020 ORGANIZAÇÃO MUNDIAL DA SAÚDE - OMS. Neurociências: consumo e dependência de substâncias psicoativas. Genebra, 2004.

PEUKER, A. C.; FOGAÇA, J.; BIZARRO, L. Expectativas e beber problemático entre universitários. Psicologia: Teoria e Pesquisa, Brasília, v. 22, n. 2, p. 193-200, 2006. http:// dx.doi.org/10.1590/S0102-37722006000200009

PILLON, S. C.; LUIS, M. A. Modelos explicativos para o uso de álcool e drogas e a prática da enfermagem. Revista Latino-Americana de Enfermagem, Ribeirão Preto, v. 12, n. 4, p. 676-682, 2004. http://dx.doi.org/10.1590/ S0104-11692004000400014

PINHO, L. B.; HERNANDÉZ, A. M. B.; KANTORSKI, L. P. Serviços substitutivos de saúde mental e inclusão no território: contradiçōes e potencialidades. Ciência, Cuidado e Saúde, Maringá, v. 9, n. 1, p. 28-35, 2010.

PINHO, P. H. et al. Reabilitação psicossocial dos usuários de álcool e outras drogas: a concepção de profissionais de saúde. Revista Escola Enfermagem da USP, São Paulo, v. 43, n. esp. 2, p. 1261-1266, 2009.

PUEL, E.; HECKRATH, M. C. R.; RIESINGER, M. C. Relatório Avaliação Centros de Atenção Psicossocial: CAPS. Florianópolis: SES; DIVE; Gerência de Atenção Básica, 2006.

RAMOS, R. T. et al. Abuso de substâncias e esquizofrenia. Revista de Psiquiatria Clínica, São Paulo, v. 24, n. 1-3/4, p. 24-27, 1997.

RIBEIRO, M.; LARANJEIRA, R.; CIVIDANES, G. Transtorno bipolar do humor e uso indevido de substâncias psicoativas. Revista de Psiquiatria Clínica, São Paulo, v. 32, p. 78-88, 2005. Suplemento 1.

SILVA, D. S.; AZEVEDO, D. M. As novas práticas em saúde mental e o trabalho no serviço residencial terapêutico. Escola Ana Nery, Rio de Janeiro, v. 15, n. 3 , p. 603-609, 2011.

SILVA, C. R. et al. Comorbidade psiquiátrica em dependentes de cocaína/crack e alcoolistas: um estudo exploratório. Aletheia, Canoas, n. 30, p. 101-112, 2009.

ZALESKI, M. et al. Diretrizes da Associação Brasileira de Estudos do Álcool e outras Drogas (ABEAD) para o diagnóstico e tratamento de comorbidades psiquiátricas e dependência de álcool e outras substâncias. Revista Brasileira de Psiquiatria, São Paulo, v. 28, n. 2, p. 142-148, 2006. 


\section{Contribuição dos Autores}

Todos os autores tiveram igual colaboração na elaboração do manuscrito enviado.

\section{Notas}

${ }^{1}$ Trata-se de um texto inédito, com apoio do Ministério da Saúde e Ministério de Ciência e Tecnologia através do edital 33/2008 do CNPq, não havendo conflitos de interesses. Trata-se de uma pesquisa aprovada pelo Comitê de Ética da Faculdade de Odontologia da Universidade Federal de Pelotas sob o n. 073/2009. 